
allemande

45-2 | 2013

Images et discours de la nation

\title{
Die Staatsoperette. Satire als Geschichtspolitik
}

\author{
Lucile Dreidemy
}

\section{OpenEdition}

Journals

Édition électronique

URL : https://journals.openedition.org/allemagne/1470

DOI : 10.4000/allemagne. 1470

ISSN : 2605-7913

Éditeur

Société d'études allemandes

\section{Édition imprimée}

Date de publication : 30 décembre 2013

Pagination : 265-282

ISSN : 0035-0974

\section{Référence électronique}

Lucile Dreidemy „Die Staatsoperette. Satire als Geschichtspolitik“, Revue d'Allemagne et des pays de langue allemande [Online], 45-2 | 2013, Online erschienen am: 29 Juli 2019, abgerufen am 20 Mai 2021 URL: http://journals.openedition.org/allemagne/1470 ; DOI: https://doi.org/10.4000/allemagne.1470 


\section{Die Staatsoperette. Satire als Geschichtspolitik}

„Kaum eine Geschichtsepoche der jüngsten österreichischen Geschichte ist für das Leservolk so vollendet unter einen passenden Hut gedrängt worden, wie die Geschichte der Ersten Republik"(1).

\section{- Lucile Dreidemy*}

Die Staatsoperette wurde am 30. November 1977 auf dem österreichischen öffentlichen Fernsehsender FS2 ausgestrahlt. Die Idee einer filmischen Aufarbeitung der tabuisierten Phase der österreichischen Zwischenkriegszeit war bereits vier Jahre zuvor zwischen dem Regisseur Franz Novotny und Verantwortlichen des ORF entstanden. Doch als Novotny, gemeinsam mit dem Komponisten Otto M. Zykan, dieses geschichtspolitisch hoch aufgeladene Thema auf dem Weg der Satire zu thematisieren beschloss, stießen sie auf heftigen Widerstand im ORF. Das Drehbuch musste neun Mal umgeschrieben, der Drehtermin viermal verschoben und die Länge des Filmes um die Hälfte gekürzt werden. Auch nach all den Kürzungen löste das endgültige Werk, bzw., mit Novotny gesprochen, das „einzige zugelassene Fragment davon“(2), einen der größten kulturpolitischen Skandale der Zweiten österreichischen Republik aus, in erster Linie durch die Hetzkampagne, die schon vor Ausstrahlung des Filmes vom katholisch-konservativen Lager initiiert wurde. In Folge des Skandals wurde der Film nie wieder ausgestrahlt und ist dadurch bis heute auch weitgehend in Vergessenheit geraten. Seine Aufnahme in die von der Filmagentur Hoanzl in Zusammenarbeit mit der Tageszeitung Der Standard und dem Filmarchiv Austria herausgegebenen DVD-Kollektion „Der österreichische Film“ 2006 konnte dieser Entwicklung nur begrenzt entgegensteuern: Mehr als dreißig Jahre nach dessen Konzipierung wirkt das damals bahnbrechende musiktheatralische Experiment zugleich verstaubt und hermetisch; für sein satirisches Wirkungspotential

* Docteur en études germaniques, ATER à l'université de Strasbourg.

1 Franz Novotny, „Aus dem Staatsoperetten Nachrichtenstudio“, in: Literaturhaus Wien, Dokumentationsstelle für neuere österreichische Literatur, Dst-Handschriftensammlung, Vorlass Franz Novotny, N1.29-3 (in der Folge: LhW/Dst/N1.29-3), Karton „Staatsoperette: Rezension, Prozess“.

2 Interview Franz Novotnys, Wien, 15.12.2011 (Aufzeichnung im Bezitz der Autorin). 
fehlt den meisten ZuschauerInnen zudem das notwendige historische Vorwissen. Nichtsdestotrotz bleibt der Film, bzw., in Novotnys Worten, das „Gesamtkunstwerk “(3), das er gemeinsam mit dem ausgelösten Skandal bildete, ein aufschlussreiches Fallbeispiel für den schwierigen Umgang der österreichischen Gesellschaft mit dem Ende der Ersten Republik und der anschließenden Diktaturerfahrung, sowie, darüber hinaus, für die Herausforderungen der Kunst im Hinblick auf einen solchen geschichtspolitischen Aufarbeitungsprozess.

Gestützt auf die wenigen bisher veröffentlichten Studien zu dem Film und auf den Vorlass des Regisseurs Franz Novotny geht diese Studie daher der Frage nach, inwiefern die Staatsoperette durch die Anlehnung an das subversive Genre der politischen Satire einen besonderen Beitrag zur Hinterfragung von gesellschaftlichen Mythen und Tabus und somit auch zur langwierigen, komplexen Reflexion über eine österreichische „Identität“ leistete. Nach einer Rekontextualisierung der Entstehungsgeschichte des Films widmet sich der Beitrag der doppelbödigen, geschichts- und kulturpolitischen Satire im Film und deren operettenhafter Umsetzung. Ein Einblick in die Entstehung des Skandals vor und nach der Ausstrahlung des Filmes rundet die Analyse ab und wirft abschließend nochmals die Frage nach den gesellschaftlichen Implikationen eines solchen künstlerischen Experiments auf.

\section{Kontext: Die sozialistische Alleinregierung und der Geist der Großkoalition}

Die Staatsoperette war eine Reaktion auf die kultur- und geschichtspolitische Lage im Österreich der 1970er Jahre. Sie wurde 1977 ausgestrahlt, also im siebten Jahr der sozialistischen Alleinregierung Bruno Kreiskys (1970-1983) ${ }^{(4)}$. Diese Phase folgte auf vier Jahre Alleinregierung der bürgerlichen Volkspartei ÖVP (1966-1970) und vor allem auf einundzwanzig Jahre Großkoalition zwischen SPÖ und ÖVP (1945-1966). Diese langjährige politische Zusammenarbeit übertrug sich auch auf den Bereich der Geschichtspolitik, indem etwa die konsenstaugliche These der geteilten Schuld der ehemaligen Bürgerkriegskontrahenten am Zusammenbruch der Demokratie in den 1920 er und 1930er Jahren zum zentralen Paradigma des offiziellen Geschichtsdiskurses der Zweiten Republik wurde. Auch die Etablierung des Fachs „Zeitgeschichte“ in der zweiten Hälfte der 1960er Jahre und die damit einhergehenden Ansätze einer differenzierteren Auseinandersetzung mit der Frage nach den Verantwortlichen für das Ende der Ersten österreichischen Republik vermochten es nicht, dieser „Koalitionsgeschichtsschreibung “(5) ein Ende zu setzen. Symbolisch für diese geschichtspolitische Kontinuität steht die Gründung 1972 der sogenannten „Wissenschaftlichen Kommission des Theodor-Körner-Stiftungsfonds und des Leopold-Kunschak-Preises zur

$3 E b d$.

4 Zur Klärung der Bezeichnung „sozialistisch“: Offiziell bezeichnete sich die sozialdemokratische Partei Österreichs von seiner Gründung 1889 bis 1945 als sozialdemokratisch, von 1945 bis 1991 als sozialistisch und seitdem wieder als sozialdemokratisch. Im üblichen Sprachgebrauch überwog allerdings auch zwischen 1945 und 1991 die Bezeichnung „Sozialdemokraten“.

5 Gerhard Botz, „Die Ausschaltung des Nationalrates und die Anfänge der Diktatur Dollfuß‘ im Urteil der Geschichtsschreibung von 1933 bis 1973“, in: Anton BenYa (Hg.), Vierzig Jahre danach. Der 4. März 1933 im Urteil von Zeitgenossen und Historikern, Wien, Doktor-Karl-Renner-Institut, 1973, S. 31-59, hier S. 40. 


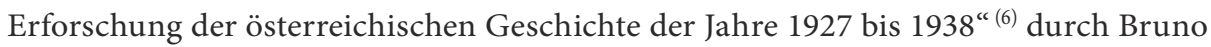
Kreisky und Nationalratspräsidenten Alfred Maleta (ÖVP), in der PolitikerInnen, JournalistInnen und HistorikerInnen aus allen politischen Lagern vertreten waren und die, so Maleta, das Ziel verfolgte, „Verständnis für die Gegner von gestern und Partner von heute zu gewinnen“"(7).

Die Kontinuität des großkoalitionären Kompromisses prägte auch den Bereich der Kulturpolitik, wo, anders als von der jungen Künstlergeneration erhofft, die alten kulturellen Strukturen und konservativen Netzwerke nicht ersetzt wurden ${ }^{(8)}$. Diese Diskrepanz hatte schon vor der Staatsoperette mehrere Skandale zur Folge; man denke etwa an die Empörungswelle, die 1975-1976 die Fernsehübertragung von Wolfgang Bauers Ibsen-Nachdichtung Gespenster, der erste Spielfilm der Medienkünstlerin Valie Export, Unsichtbare Gegner, sowie Peter Turinis und Wilhelm Pevnys Fernsehserie Alpensaga bereits ausgelöst hatten. So gesehen war der Skandal um die Staatsoperette, mit der Literaturwissenschaftlerin Evelyne Polt-Heinzl gesprochen, „nur die Spitze des kulturpolitischen Eis(zeit)berges“ der 1970er Jahre ${ }^{(9)}$.

Um diese hartnäckigen geschichts- und kulturpolitischen Deutungsmuster zu durchbrechen und einen Prozess des Nach- und Umdenkens in der Gesellschaft zu fördern, beschloss Novotny, sich von dem herkömmlichen Dokumentarfilm zu distanzieren, zugunsten eines experimentelleren Formats, das „die Urteilsbildung des Seherpublikums anzuregen“"vermöge ${ }^{(10)}$. Das Ziel von Novotny und Zykan lag jedoch von Anfang an nicht darin, den Protagonisten der Zwischenkriegszeit einen posthumen Prozess zu machen. Dementsprechend hieß es auch an einer Stelle des vorläufigen Drehbuchs des Films: „Übrigens: Der Faschismus lässt sich nicht mit Witz und Ironie bekämpfen... “(11). Dabei knüpften die Künstler an die Reflexion ihres Vorbilds, Charlie Chaplin, an, der rückblickend das Konzept seines Films The Great Dictator grundsätzlich in Frage stellte, weil solche Verbrechen nicht mit Humor behandeln werden könnten ${ }^{(12)}$. Im Unterschied zu Chaplin war daher ihr Anspruch von Anfang an geschichtspolitisch und zielte darauf $a b$, die Unzulänglichkeiten des gegenwärtigen offiziellen Geschichtsdiskurses

6 Ernst Hanisch, „Die Dominanz des Staates. Österreichische Zeitgeschichte im Drehkreuz von Politik und Wissenschaft“, in: Alexander Nuetzenadel, Wolfgang Schieder (Hg.), Zeitgeschichte als Problem. Nationale Traditionen und Perspektiven der Forschung in Europa, Goettingen, Vandenhoeck \& Ruprecht, 2004, S. 54-77, hier S. 58.

7 Maleta, zit. nach Ludwig Jedlicka, Rudolf Neck (Hg.), Vom Justizpalast zum Heldenplatz, Studien und Dokumentationen 1927 bis 1938, Wien, Verlag der Österreichischen Staatsdruckerei, 1975, S. 8.

8 Evelyne Polt-Heinzl, „Kulturskandale der 1970er Jahre. Lauter kleine Staatsoperetten“, in: Ders. (Hg.), Staatsoperetten. Kunstverstörungen. Das kulturelle Klima der 1970er Jahre, Wien, Dokumentationsstelle für Neuere Österreichische Literatur, 2009, S. 9-42, hier S. 12.

9 Ebd., S. 18.

10 Interview Novotnys über das Konzept des Films, nachzulesen im Artikel „Österreichische Vergangenheit“, Profil, 29.6.1976, LhW/Dst/N1.29-3, Karton „Staatsoperette: Rezension, Prozess“, Mappe „Staatsoperette und Erklärung der Autoren, Rez. 1977“.

11 Zit. nach: LhW/Dst/N1.29-3, großformatiges Manuskript der Staatsoperette.

12 „Had I known of the actual horrors of the German concentration camps, I could not have made The Great Dictator, I could not have made fun of the homicidal insanity of the Nazis." Charles ChapLin, My autobiography, New York, Simon and Schuster, 1964, S. 392. In dem Interview am 15. Dezember 2011 bezog sich Novotny ausdrücklich auf diese Reflexion Chaplins. 
bloßzustellen. Das angebrachteste Format dafür fanden sie im Genre der Operette wieder. In diesem Sinne hielt Novotny in seinen konzeptuellen Reflexionen fest: „Man soll auch nicht übersehen, daß die aggressive Aufbereitungsform als ,Staatsoperette dann elementar notwendig wird, wenn man den beschwichtigenden Werken, die sich mit der Zeit befassen, begegnen will“(13).

Über die großkoalitionäre Geschichtsschreibung hinaus wandten sich die Künstler in erster Linie gegen die Dominanz des konservativen Geschichtsbildes in der österreichischen Geschichtspolitik. Aus diesem Grund vermerkte Novotny in seinen Notizen, Bezug nehmend auf eine jüngste Parlamentsdebatte über den Bundeskanzler und Diktator Engelbert Dollfuß: „Daß sich damals die großbürgerliche Partei nicht endlich von Dollfuß distanziert hat, sondern ganz gegenteilig für ihn parlierte, hat mir die Notwendigkeit der provokativen Geschichtsdarstellung in der Staatsoperette bewiesen “ (14). Dieser Provokationsdrang spiegelt zugleich das Aufklärungsbedürfnis jener jungen Nachkriegsgeneration (Novotny war 1949 geboren) wider, die den Austrofaschismus nicht mehr erlebt hatte und auch in der Schule davon nichts erfuhr, da die GeschichteSchulbücher bis zum Zeitpunkt der Ausstrahlung des Films 1977 die Dollfuß-/Schuschnigg-Diktatur kaum thematisierten. Dementsprechend begründeten Novotny und ORF-Redakteur Preiner die beabsichtigte Ironisierung der historischen Begebenheiten als Antwort auf die bisherige Tabuisierung dieser zeithistorischen Periode ${ }^{(15)}$.

Die Staatsoperette arbeitete ironisch dekonstruktiv, schrieb sich aber gleichzeitig in eine Tradition ein, die Operettentradition. Novotny und Zykan griffen das „Nationalheiligtum Operette “(16) bewusst wieder auf, um das Genre des rührseligen, gutbürgerlichen Musiktheaters zu verfremden, und in den Dienst der satirischen Bloßstellung von gesellschaftlichen Tabus zu stellen. Diese Verfremdung war insofern nur teilweise eine solche, als sich die Künstler, indem sie sich von der harmlosen Nostalgie und Walzerseligkeit der Wiener Operette distanzierten, gleichzeitig an die ursprünglichen Ansprüche der Operette Offenbacher Tradition wieder anknüpften, die in der ersten Hälfte des 19. Jahrhunderts als entscheidendes Medium politischer Persiflage fungierte. Mit dieser Form der provokativen Hinterfragung von Tabus und Mythen trafen Novotny und Zykan auf das Interesse einer anderen charismatischen Vertreterin dieser neuen, kritischen Generation österreichischer KünstlerInnen und Intellektueller, nämlich Elfriede Jelinek, die über die Staatsoperette festhielt: „Es gibt in Österreich nicht so viele Filmemacher, die etwas mit Satire anfangen können. Ich hab' jemanden gesucht, der nicht mit differenzierenden, psychologisierenden, filmästhetischen

13 Typoskript Novotnys, LhW/Dst/N1.29-3, Karton „Staatsoperette: Rezension, Prozess“, Mappe „Staatsbegräbnis - Staatsoperette“.

14 Ebd. Novotny spielt hier höchstwahrscheinlich auf die Parlamentsdebatte im März 1973 an, als ÖVP-Parlamentarier, die vom jungen SPÖ-Politiker Heinz Fischer aufgrund der Beibehaltung eines Dollfuß-Porträts in deren Parlamentsklub angegriffen worden waren, sich ausdrücklich für Dollfuß einsetzten. Vgl. Stenographisches Protokoll der 69. Sitzung des Nationalrates der Republik Österreich, XIII. Gesetzgebungsperiode, 4.4.1973, S. 6553-6555.

15 „Österreichische Vergangenheit“, Profil, 29.6.1976, LhW/Dst/N1.29-3, Karton „Staatsoperette: Rezension, Prozess“, Mappe „Staatsoperette und Erklärung der Autoren, Rez. 1977“.

16 Carsten FAstner, „Satire oder Sentiment?“, Falter, 13.4.2005, URL: http://www.falter.at/falter/2005/ 04/12/satire-oder-sentiment/ (letzter Zugriff: 4.10.2013). 
Mitteln arbeitet, sondern, eher wie ich, mit einer holzschnittartigen Schwarzweißtechnik in Richtung starker Kontraste “(17).

\section{Vom Künigelberg zum Zauberberg: Die hindernissreiche Entstehungs- geschichte des Films und deren satirische Aufarbeitung im Film}

Das Projekt begann 1972 auf Anregung von Hans Preiner, dem damaligen Leiter der ORF-Dokumentarsendereihe Impulse, für welche Novotny bereits diverse (Kurz-)Filme produziert hatte. Aus Preiners Vorschlag eines Beitrags zum sogenannten PfrimerPutsch 1931 entstand 1973 das Projekt Staatsbegräbnis, das aber schon weit mehr als den von Walter Pfrimer, dem Landesleiter des Steirischen Heimatschutzes, 1931 initiierten Staatsstreich behandelte, und sich bereits deutlich vom klassischen Format des Dokumentarfilms unterschied ${ }^{(18)}$. Der ORF wurde Träger und Produzent des Fernsehprojekts und finanzierte es mit vier Millionen Schilling, das BMUK (Bundesministerium für Unterricht und Kunst) subventionierte es mit etwa einer Million. Die Verantwortlichen im ORF versuchten jedoch von Anfang an, die künstlerische Tätigkeit in gewisse Bahnen zu lenken.

Der erste Drehtermin war für September 1975 geplant. Bevor dies zustande kam, wurde Novotny jedoch seitens des ORF nahegelegt, den Produktionstermin bis zur Fertigstellung des neuen ORF-Fernsehtheaters auf dem Wiener Künigelberg zu verschieben. Parallel dazu hatte der Komponist Otto M. Zykan die Partitur für das Stück in ein Großprojekt umgeschrieben, das nun das achtzig Musiker starke ORF-Symphonieorchester beanspruchte. Durch diese logistischen und konzeptuellen Änderungen stiegen die Produktionskosten von geplanten sechs auf fast neun Millionen Schilling an und das Projekt wurde in die Länge gezogen. Daraufhin sprach der Intendant des Fernsehkanals FS 1 und spätere ORF-Generalintendant Gerhard Weis in einer öffentlichen Presseaussendung im Januar 1976 von einer „Kostenexplosion“ und nahm diese Situation zum Anlass, um die weitere Vorgangsweise des ORF von der Kompromissbereitschaft der Künstler abhängig zu machen und eine Absetzung der Produktion in den Raum zu stellen. Diese Drohungen wurden allerdings kurze Zeit später von Weis als „Missverständnis“ relativiert ${ }^{(19)}$.

Nach einer Neuaufteilung der Unterhaltungsbranche des ORF im April 1976 übernahm der Intendant von FS2, Franz Kreuzer, die Verantwortung für das nun Staatsoperette genannte Filmprojekt und setzte sich von nun an für dessen Verwirklichung ein. Nichtsdestotrotz hielt der Druck seitens des ORF an: Nach mehrmaliger Erhöhung der Produktionskosten und neunmaliger Verschiebung begannen die Dreharbeiten erst am 22. März 1977; die ursprünglich geplante Drehzeit von anderthalb Monaten wurde auf vier Tage und die Spieldauer von 90 auf 35 Minuten gekürzt.

17 Elfried JelineK, in: Volksstimme, 15.10.1982, zit. nach Irene Suchy, „Das Werden der Staatsoperette in Originaldokumenten. Rekonstruktion eines Prozesses“, in: E. Polt-Heinzl (Hg.), Staatsoperetten (Anm. 8), S. 86-122, hier S. 90.

18 Vgl. die ersten Versionen des Drehbuchs, LhW/Dst/N1.29-3, Karton „Staatsoperette: Gesamtprojekt“, Mappe „Staatsbegräbnis-Entwürfe 1973/74“.

19 Vgl. anonyme Zeitungsnotiz „Staatsoperette“, Arbeiter Zeitung, 10.3.1976, S. 7. 
Auch wenn dadurch etliche Passagen gestrichen werden mussten, beschlossen die Künstler kurz vor der Ausstrahlung, den politischen Druck, der sie bei der Konzeptualisierung des Films begleitet hatte, in das Szenario einzubeziehen und satirisch bloßzustellen. Zu diesem Zweck wurde die eigentliche Operette - die in der endgültigen Version des Filmes immerhin 55 statt den vorgesehenen 35 Minuten dauerte - um eine zehnminütige Rahmengeschichte erweitert ${ }^{(20)}$. Diese Anfangs- und Schlussszenen spielen in den 1970er Jahren und handeln von den vergeblichen Verhandlungen des Autors „Bamberger“ mit eifrigen Vertretern der offiziellen Kulturpolitik.

Die Diskussionen beginnen in einem Stiegenhaus und führen dann in einen Konzertsaal; die Assoziierung mit dem Wiener „Musikverein“(21) ist eindeutig. Vor dem ersten Dialog im Stiegenhaus setzt jedoch die Anfangsszene, und somit auch der Film, mit einer vollkommen anderen Szenerie an, nämlich der längeren Aufnahme eines Bergschlosses bei Abenddämmerung. Über diese subtile Anspielung auf Thomas Manns Zauberberg wird der ORF und somit auch die Kulturpolitik der 1970er Jahre als abgekapselte, realitätsferne Welt dargestellt. Über die Anspielung auf das Sanatorium Berghof werden die Bürokraten darüber hinaus mit dubiosen Therapeuten gleichgesetzt, die mit engstirnigen Anweisungen versuchen, einen irregeleiteten Künstler auf den richtigen Weg zu bringen. Dementsprechend setzt dann auch die erste Dialogssequenz mit der oberlehrerhaften Empfehlung eines der Bürokraten an: „San's doch g'scheit. Ein paar Striche im Text, wir wollen Ihnen nicht vorschlagen wo, und alles in scho' vü leichter“(22). Dadurch weist die Szene sofort unmissverständlich auf den politischen Druck und die zensurähnlichen Hindernisse hin, denen Zykan und Novotny in den vier Jahren der Entstehung des Films ausgesetzt waren. Streichungen, Erhöhungen der Produktionskosten und mehrmalige Verschiebungen der Produktionstermine bleiben auch in der Folge die Hauptthemen der Verhandlungen, die im Stil Loriots ${ }^{(23)}$ vor allem die Engstirnigkeit und Kommunikationsunfähigkeit des kulturpolitischen Establishments zum Ausdruck bringen. Im Konzertsaal schaltet sich, nicht von ungefähr vor dem Hintergrund von Frederic Chopins Marche Funèbre, ein leitender Manager in die Diskussion ein: „In ihrem Projekt hat es das letzte Mal Zusammenhänge gegeben, die wir nicht verantworten konnten“(24), wirft er Bamberger vor, und der erste Bürokrat fügt mit einem herablassenden Lachen hinzu: „Ja, hoffentlich hängt nicht wieder das eine mit was anderem zusammen. Hier herein gehören nur Sachen, die unter einen einzigen Hut passen!“ (25). Der hier bereits angedeutete Zynismus gipfelt am Ende der Anfangsszene in der Reflexion des zweiten Bürokraten: „Auch unsere Bürokratie kann, wie die

20 Gerhard Pistor, „Mühsame Geburt der TV-Operette“, Kurier Fernsehen, 4.2.1977, LhW/Dst/N1.29-3, Karton „Staatsoperette: Rezension, Prozess“, Mappe „Staatsoperette und Erklärung der Autoren, Rez. $1977^{\prime}$.

21 Drehbuch Staatsoperette (Zwischenspiele zur Fernsehfassung), LhW/Dst/N1.29-3, Karton „Staatsoperette: Gesamtprojekt“". Zit. nach F. Novotny, Die Staatsoperette, ORF 1977, Hoanzl 2006, DVD, 67 min, hier 00:44-00:52. Vgl. Wolfgang Gratzer, „Die Staatsoperette, ein signifikanter Skandal“, in: Peter Csobádi, Gernot Gruber (u. a.) (Hg.), Politische Mythen und nationale Identitäten im (Musik-)Theater, Anif/Salzburg, Verlag Mueller-Speiser, 2003, Bd. II, S. 890-903, hier S. 892. 
Kunst, plötzlich und direkt wirken. Oder soll es eine Kunst geben, die ohne Bürokratie leben kann? Eine Bürokratie ohne Kunst gibt es schon, aber... “(26). Dieses Fazit verstärkt nochmals den Eindruck, dass die Bürokraten über ihr starrsinniges und zynisches Verhalten gegenüber dem vorgestellten Projekt vor allem sich selber bzw. die Kulturpolitik, die sie vertreten, in Frage stellen ${ }^{(27)}$. Somit liefern diese kurzen Rahmensequenzen eine ungeschliffene Parodie des Subventionsstaats, der die Regeln des künstlerischen Schaffens diktiert ${ }^{(28)}$.

Die Schauspieler dieser Rahmengeschichte sind bis auf wenigen Ausnahmen auch die Akteure der eigentlichen Operette. Ernst Meister beispielsweise, der in der Operette den Prälaten Ignaz Seipel, bzw. „Schwarz“ spielt, gibt in den Rahmensequenzen den tonangebenden Manager. Die Parallelität, bzw. Kontinuität zwischen beiden Figuren wird durch die Beibehaltung des predigthaften Tons des Geistlichen signalisiert ${ }^{(29)}$. Auch wenn die Parallelität - wahrscheinlich aufgrund der knappen verfügbaren Zeit für die Konzeption der Zwischenspiele - meist nur beim genauerem Hinsehen erkennbar wird, bleibt dieses dramaturgische Kunststück von besonderem Interesse als parabelhafte Anspielung auf die nach 1945 etablierten Politik des Kompromisses zwischen den ehemaligen Bürgerkriegskontrahenten. Über diesen Kunstgriff wird bereits die zweite Ebene der Kritik im Film angedeutet, nämlich die geschichtspolitische Satire.

\section{Die Satire als Instrument der Hinterfragung geschichtspolitischer Tabus}

Der Hauptteil des Filmes besteht aus einer satirischen Darstellung der österreichischen Zwischenkriegszeit. Auch wenn die Parallele mit historischen Ereignissen und Akteuren nie ausgesprochen wird, lassen bekannte Charakterzüge und historische Elemente die politische Entwicklung zwischen 1927 und 1934 unmissverständlich als die historische Folie des Szenarios erkennen ${ }^{(30)}$.

26 Zit. nach ebd., 06:12-06:28.

27 Drehbuch Staatsoperette (Zwischenspiele zur Fernsehfassung), LhW/Dst/N1.29-3, Karton „Staatsoperette: Gesamtprojekt“.

28 Vgl. dazu auch Sabine Zelger, „Die Störung des nationalen Blicks oder Warum der österreichische Bildungskanon nicht auf die Staatsoperette verzichten darf“, in: E. Polt-Heinzl (Hg.), Staatsoperetten (Anm. 8), S. 123-134, hier S. 127.

29 Dabei wurde zudem mit dem Erkennungszeichen von Meisters prägendem Timbre gespielt, das dem zeitgenössischen Publikum über die wöchentliche, populäre und von Meister gesprochene Fernsehsendung „Der Fenstergucker“ bekannt war. Vor diesem Hintergrund sahen die Künstler einen besonderen Witz darin, ausgerechnet einen der berühmtesten Fernsehsprecher Österreichs, oft als „die Stimme Österreichs“ bezeichnet, für das satirische Projekt zu gewinnen. Vgl. Interview Franz Novotnys, Wien, 15.12.2011.

30 In den früheren Versionen des Drehbuchs wurde auch die Entwicklung des austrofaschistischen Regimes nach Dollfuß' Tod thematisiert und insbesondere Schuschniggs Persönlichkeit und Politik, unter anderem seine Unterwürfigkeit gegenüber Hitler - bzw. „Stiefel“ oder „Wolf“, so Hitlers Name im Drehbuch - parodiert. Schuschnigg, bzw. „Strumpf“, „Schussl“ oder „Grau“ im Drehbuch, trat meist als ahnungsloser Mitläufer von Schwarz in der Uniform eines Internatszöglings mit Schulranzen, Matrosenmütze und Ärmelschonern auf. Eine detaillierte Szene wurde unter anderem dem Besuch von Grau - mit Pudel - beim „Herrn Oberwolf“ und seinen zehn Schäferhunden gewidmet - eine unmissverständliche Parodierung des Berchtesgadener Treffens. Vgl. LhW/Dst/N1.29-3, großformatiges Manuskript der Staatsoperette. 
Die Handlung setzt unter der Kanzlerschaft des christlichsozialen Bundeskanzlers Ignaz Seipel (1926-1929) an. Prägend für die zweite Amtszeit des Prälaten und christlichsozialen Politikers war die Gründung eines Bürgerblocks bestehend aus Christlichsozialen, Großdeutschen und Landbund zur Bekämpfung der Sozialdemokratie. Die spontane Revolte der sozialdemokratischen Basis und der Brand des Justizpalastes am 15. Juli $1927^{(31)}$ brachten sowohl die Brutalität dieser Politik als auch die Zersplitterung der Sozialdemokratie zwischen dem Widerstandsdrang der Basis und den Besänftigungsversuchen der Spitze zum Ausdruck. In der Folge radikalisierte Seipel nochmals seine antimarxistische Politik, indem er sich zunehmend der faschistischen Miliz der Heimwehr annäherte ${ }^{(32)}$. Dieser politische Kurs wurde nach Seipels Amtszeit weitergeführt und vor allem ab der Machtübernahme des christlichsozialen Bundeskanzlers Engelbert Dollfuß 1932 verstärkt. An der Spitze einer Koalition zwischen Christlichsozialen, Landbund und dem politischen Arm der Heimwehr, dem „Heimatblock“, verfolgte Dollfuß einen antimarxistischen, antipluralistischen und antiparlamentarischen Kurs und ließ Schritt für Schritt alle Grundlagen des demokratischen Rechtsstaates abschaffen. Außenpolitisch holte sich das Regime Unterstützung beim faschistischen Italien. Diese Entwicklung ging mit einer stärkeren Repression der Opposition einher, führte zur Wiedereinführung der Todesstrafe und gipfelte im Februar 1934 in einem Bürgerkrieg.

Das Satirische der Operette ergibt sich großteils aus der überspitzten Parodierung der Hauptakteure dieser Phase. Seipel wird als der machtbesessene Prälat und Sozialistenhasser „Schwarz“ porträtiert. Hauptprotagonist der Sozialdemokraten ist der vollkommen überforderte Arbeiterführer „Griffel“, eine ebenso gnadenlose Verulkung des sozialdemokratischen Parteivorsitzenden und Theoretikers Otto Bauer in der Figur eines ängstlichen Intellektuellen auf der verzweifelten Suche nach einem Konsens mit den Machthabern und in panischer Angst vor dem Wort „Revolution“. In Italien besucht Schwarz „Muffolini“, einen selbstverliebten „Macho“ umgeben von nackten Gespielinnen, der sich trotz seiner antikatholischen Einstellung darauf einlässt, den Prälaten mit Waffen für den Kampf gegen die Sozialdemokratie auszustatten. Dem Operettenformat entsprechend interagieren die Figuren nicht nur sprechend, sondern versuchen sich auch singend und tanzend verständlich zu machen. Die dadurch ins Absurde abgleitenden Dialoge bilden eines der Hauptvehikel der Satire.

Besonders ersichtlich werden die satirischen Ansprüche der Künstler und deren Umsetzungstechnik auch in der Parodierung von Dollfuß' Machtübernahme: Als Schwarz den wachsenden Widerstand der linken Opposition verzweifelt betrachtet und Gott um Hilfe bittet („Ich brauche einen Mandatar, einen Mann der Tat“, schreit er mit gehobener Faust, „der's in den Armen hat, mit festem Schritt und Tritt“ (33)),

31 Diese spontane Revolte folgte dem Freispruch von zwei Mitgliedern der antimarxistischen paramilitärischen „Frontkämpfervereinigung“, die sechs Monate zuvor anlässlich einer friedlichen sozialdemokratischen Demonstration im burgenländischen Schattendorf einen älteren Mann und ein Kind erschossen hatten. Die Massendemonstration in Wien wurde von der Polizei niedergeschlagen und endete mit 89 Toten und dem Brand des Justizpalastes. Vgl. Gerhard Bотz, Gewalt in der Politik. Attentate, Zusammenstöße, Putschversuche, Unruhen in Österreich 1918 bis 1938, München, Wilhelm Fink Verlag, 1983, S. 308.

32 Besonders augenscheinlich wird diese Annäherung in Ignaz Seipels Tübinger Rede zur Kritik der Demokratie, am 15. Juli 1929, nachzulesen in der Reichspost vom 18. Juli 1929, S. 8 f. 
kündigt ein tiefer Blechton das Unerwartete an: Vom Himmel schwebt langsam, als wäre er schwerelos, ein großer Geschenkkarton mit rosa Schleife zu Boden, aus dem eine zwergengroße Figur auf einem Einrad herausfährt. Am Rücken des „possierlichen Spielzeuges“ “(34) dreht sich ein überdimensionaler Schlüssel zum Aufziehen eines Federwerks - ein bekanntes Motiv aus der Karikaturwelt ${ }^{(35)}$. Der Zwerg trägt einen schwarzen Frack mit Fliege und offizieller Kanzlerschärpe, und am Kopf einen Zylinder. Diese Attribute und die kleine Statur verweisen unzweifelhaft auf den 1,53 m großen Dollfuß. Die Parodie der göttlichen Postsendung, überspitzt im unerwarteten Auftritt des Zwerges in Form eines ironisch neuaufgelegten Deus ex machina-Effekts, dekonstruieren das in den 1930er Jahren propagierte Bild Dollfuß' als Messias, den Gott gesandt habe, um Österreich aus dem Chaos zu retten. Die aus der Zirkuswelt bekannten Attribute des Zylinders und des Einrads tragen nochmals dazu bei, das Motiv des göttlichen Messias ins Lächerliche zu ziehen. Die unbeholfene Bewegung des Hampelmanns und seine Fernsteuerung spielen wiederum auf ein bekanntes Deutungsmuster der konservativen Geschichtsschreibung an, nämlich die Idee, dass die brenzliche innenund geopolitische Lage Österreichs den kleinen Bundeskanzler des kleinen Österreichs dazu gezwungen hätten, den Anweisungen von mächtigeren Dritten zu folgen, in erster Linie des faschistischen „Duce“, Mussolini.

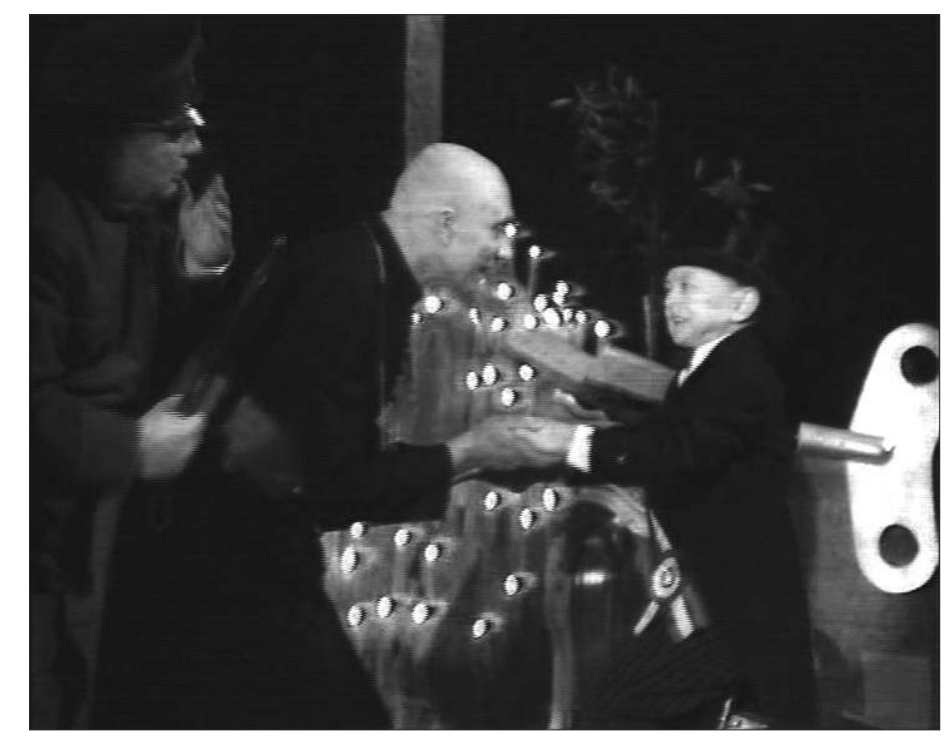

Schwarz trifft Hackl (Quelle: Die Staatsoperette, ORF 1977, Hoanzl 2006, DVD) ${ }^{(36)}$

34 Zit. nach: LhW/Dst/N1.29-3, großformatiges Manuskript der Staatsoperette.

35 Dollfuß wird vom 1,14 m großen Schauspieler Fritz Hackl gespielt, der im Burgtheater üblicherweise Zwerge und Kinder darstellte. Die Verwendung des Einrads ergab sich womöglich erst aus der Zusammenarbeit mit Hackl, der seinen Lebensunterhalt vor dem Burgtheater mit Kunststücken auf einem Einrad im Wiener Prater verdiente. Vgl. Interview Franz Novotnys, Wien, 15.12.2011.

36 Herzlichen Dank an Franz Novotny für die Erlaubnis, Screenshots aus seinem Film abzudrucken. 
Auf die Frage des verdatterten Prälaten: „Kleiner, willst du Kanzler werden?“ (37), antwortet „Hackl“ - so der Name des Zwergs im Drehbuch ${ }^{(38)}$ - mit schnarrender, selbstbewusster Stimme: „Ich werde nicht nur Kanzler werden, sondern auch das liberalkapitalistische Wirtschaftssystem und den Einfluss der roten Banden, bzw., wenn es sein muss, vielleicht sogar, das Parlament, ausschalten!“, und stellt daraufhin sein politisches Programm in Form eines Wiener Liedes vor. Bereits die ersten Parolen: „I’ bin so g'sund / Mi' beißt ka Hund / I' bin auf alles g'fasst /“ und daraufhin „Meine Hochverehrte Damen und Herren! / Ich hoffe doch sehr, sie haben mich noch gern!“(39), gesungen mit unbekümmerter, froher Stimme, kontrastieren das Bild der lächerlichen Zirkusfigur und des ferngesteuerten Hampelmanns, indem sie eine sehr selbstbestimmte Persönlichkeit erkennen lassen. In der zweiten Strophe wird die Tonalität des Liedes etwas düsterer und Hackl ernster: „Ach wos! Es wird sicher nix g'scheh'n / I' bin auf alles g'fasst / Ihr werdet seh'n, der Tod ist schön / Auch wenn's euch gar net passt! / Und stirbt das Volk für seinen Kanzler hin / Ja dann hat mein Leben erst einen richtigen Sinn!“(40). In diesen Sätzen werden viele Charakteristika von Dollfuß’ Persönlichkeit wiederaufgegriffen, wie etwa sein starkes Selbst- und Sendungsbewusstsein, aber auch die Verbrechen seines Regimes in Erinnerung gerufen, in erster Linie im Hinblick auf den Bürgerkrieg im Februar 1934, als die Regierung Dollfuß die Widerstandsaktion von SozialdemokratInnen und KommunistInnen mit Einsatz der Artillerie niederschlagen, und führende Persönlichkeiten der Opposition standrechtlich hinrichten ließ.

Diese Szene bietet zugleich einen guten Einblick in die Rolle der Musik im Film. Zykans Komposition, ein aufwendiges Werk für Orchester, wurde bewusst zur Verstärkung der satirischen Bildwirkung eingesetzt. Durch die operettenhafte Kombination des oberflächlichen Tons des Volkslieds und dessen kaltblütiger Botschaft trägt das Wiener Lied dazu bei, Dollfuß' Selbstinszenierung als volksnaher Führer zu verfremden ${ }^{(41)}$. Der zynische Ton des „kleinformatigen Führers österreichischer Prägung“ (42), so das Drehbuch, verstärkt das durch seine Inszenierung als Geschenk Gottes bereits erweckte Gefühl der Abgehobenheit des Politikers. Somit wird die in den 1960er und 1970er Jahren noch verbreitete Einschätzung Dollfuß' als demokratisch gesinnten Politiker $^{(43)}$, der nur aufgrund des innen- und geopolitischen Drucks zu autoritären Maßnahmen gezwungen worden sei, grundsätzlich delegitimiert.

37 Zit. nach F. Novotny, Die Staatsoperette (Anm. 22), 50:49-51:09.

38 Nachdem er zunächst zum Spitznamen „Dollbein“ tendiert hatte, kam Novotny letztlich zum Namen „Hackl“ zurück. Zu „Dollbein“, vgl. LhW/Dst/N1.29-3, Karton „Staatsoperette: Gesamtprojekt“, Mappe „Unikate. Arbeitsunterlagen: ,Staatsbegräbnis““.

Zit. nach F. Novotny, Die Staatsoperette (Anm. 22), 51:20-51:45.

Zit. nach $e b d$., 52:09-52:36.

41 Neben dem Wiener Lied werden in Zykans tonaler Musik sämtliche musikalische Klischees, vom Gregorianischen Choral, über Walzer und Schlager, und bis zu sozialistischen Hymnen, eingesetzt und verfremdet. Vgl. dazu: I. Suchy, „Das Werden der Staatsoperette in Originaldokumenten“ (Anm. 17), S. 91. Aus diesem Werk machte Zykan 2000 eine Konzertversion, die sich allerdings in mehrfacher Hinsicht von der Filmmusik abhebt. Vgl. W. Gratzer, „Die Staatsoperette“(Anm. 23), S. 902.

42 Zit. nach: LhW/Dst/N1.29-3, Karton „Staatsoperette: Gesamtprojekt“, Mappe „Vom Pfreimer Sterz zur ,Staatsoperette', 8 Stadien des Stoffes bis zu seinem Verbot“.

43 Vgl. dazu Hugo Hantsch, „Engelbert Dollfuß (1892-1934)“, in: Ders. (Hg.), Gestalter der Geschicke Österreichs, Innsbruck/Wien/München, Tyrolia-Verlag, 1962, S. 611-623, hier S. 611. Anlässlich eines 
Über die musikalische Komponente hinaus dient das Genre der Operette zur Abstraktion und zur Verdichtung der Handlung und macht aus dem musiktheatralischen Werk eine komplexe, vielschichtige Parabel in der Tradition von Bertolt Brechts epischem Theater ${ }^{(44)}$. Das Abstraktionsverfahren ermöglicht insbesondere den Künstlern, mit Anachronismen zu spielen und mehrere historische Figuren in einer zu porträtieren. Besonders ersichtlich wird dieses typische Verdichtungsverfahren an der Folgeszene, nämlich der Szene von Schwarz’ Besuch bei Muffolini, die zugleich die Abschlussszene der Operette darstellt.

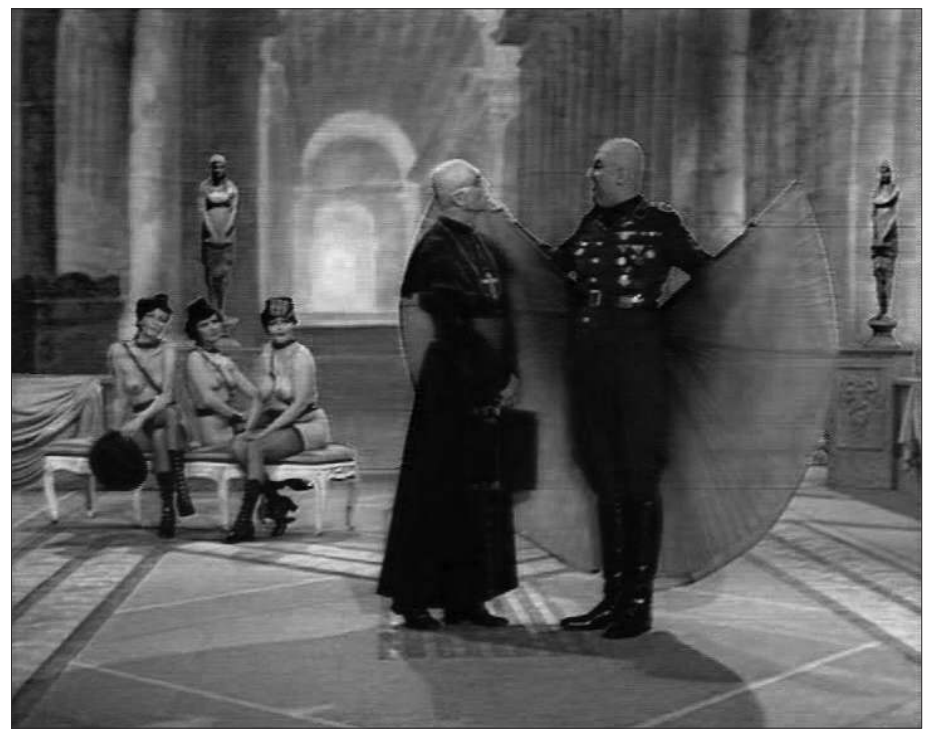

Schwarz trifft Muffolini (Quelle: Die Staatsoperette, ORF 1977, Hoanzl 2006, DVD)

Tatsächlich fand nie ein Treffen zwischen Seipel und Mussolini statt. Wohl aber reisten Heimwehrführer Starhemberg 1932 und Dollfuß 1933 zum „Duce“. Warum wird also im Film ausgerechnet ein Rom-Besuch von Schwarz inszeniert? Eine mögliche Erklärung liegt darin, dass dem Provokationsanspruch des Films kaum effizienter entsprochen werden konnte als durch die kontrastreiche Inszenierung des Geistlichen in Kutte in der erotischen Welt von Muffolini. Zugleich wird aus dem Operettenprotagonisten Schwarz eine komplexe Figur, in der sich mehrere historische Persönlichkeiten verschränken, nämlich

Festkommers des Cartellverbandes in Wiener Neustadt am 25. Mai 1968 bezeichnete auch der ÖVPBundeskanzler Joseph Klaus Engelbert Dollfuß als „makellose[n] Demokrat[en]“. Vgl.: „Klaus bricht Lanze für Dr. Dollfuß“, Kurier, 27.5.1968, S. 2.

44 In manchen früheren Versionen ist die Anlehnung an das epische Theater noch ausgeprägter, wie etwa durch die reflexive Distanzierung der Figuren von ihrer Rolle. Als etwa in einem Drehbuch ein Streit zwischen Schwarz, Hackl und „Bräunel“, Karikatur des zwielichtigen, christlichsozialen und NS-nahen Anton Rintelens, beginnt, unterbricht Bräunel mit folgendem Hinweis: „Aber ich bitte Sie, meine Herren. Nehmen Sie ihre Rollen nicht allzu ernst. Ich bin der Schauspieler X, Sie sind Herr X und Sie Herr X; wenn die Szene abgedreht ist, sind wir wieder gute Freunde und prosten uns zu." Zit. nach: LhW/Dst/N1.29-3, Karton „Staatsoperette: Gesamtprojekt“, Mappe „Vom Pfreimer Sterz zur ,Staatsoperette', 8 Stadien des Stoffes bis zu seinem Verbot“. 
Dollfuß, Starhemberg und Seipel. Durch dieses dramaturgische Kunststück wird es nun möglich, mit einer Szene auf zwei historische Ereignisse gleichzeitig anzuspielen: Dollfuß' Besuche in Rom ab April 1933 und auch Starhembergs Besuch bei Mussolini 1932, der 1933 zur sogenannten „Hirtenberger Waffenaffäre“, einer Waffenlieferung Italiens an die Heimwehr, führte. Dieses Abstraktions- und Verdichtungsverfahren manifestiert sich auch an einer anderen Stelle der Operette, nämlich in der Sequenz über die Hinrichtung des „Fürsten“ auf Anweisung von Schwarz. Auch hier dient eine fiktive Szene dazu, über die Verschränkung von zwei historischen Figuren, diesmal Seipel und Schuschnigg, eine Parabel der Entmachtung der Heimwehr durch Kurt Schuschnigg im Jahre 1936 zu erzeugen. Diese Technik der Parabel setzt auf das historische Vorwissen der ZuschauerInnen und animiert sie dazu, fiktive Darstellungen im Film von historischen Begebenheiten zu unterscheiden. Somit wird die bewusste Geschichtsklitterung ${ }^{(45)}$ zum Vehikel der Bloßstellung von tabuisierten historischen Begebenheiten, wie etwa hier von Dollfuß' politischen und persönlichen Verbindungen zum faschistischen Führer Mussolini.

Über diese Konfrontation der ZuschauerInnen mit Vieldeutigkeiten und Illogismen zielten die Künstler darauf ab, das zeitgenössische Publikum zum Nachdenken und zum Misstrauen aufzufordern - auch hier wieder ganz in Brechtscher Tradition. In diesem Sinne schrieb Novotny über die Staatsoperette: „Ein Stück wie die Staatsoperette will (sofern ein Stück wollen kann) der Entpolitisierung und Leitbarkeit des Publikums entgegenwirken. Indem es zu den herkömmlichen Geschichtsdarstellungen einen wirklichen Kontrapunkt setzt und die meinungslose Ausgewogenheit hintanstellt, reißt es natürlich auch den Liberalen vom Sessel und zwingt ihn in die Diskussion, der er sich sonst entziehen kann“(46).

Trotz dieser Ansprüche überstieg der Skandal, der den Film hervorrief, die Erwartungen der Künstler bei weitem ${ }^{(47)}$.

\section{Der Skandal}

Ein Charakteristikum des Skandals um die Staatsoperette besteht darin, dass er schon vor der Ausstrahlung des Filmes initiiert und zum Teil durchgeführt wurde. So bezieht sich etwa mehr als ein Drittel der 140 Seiten schweren Pressesammeldokumentation zu der Hetzkampagne auf die Zeit vor der Ausstrahlung ${ }^{(48)}$.

Bereits im März 1977 widmete der Kurier, Österreichs zweitgrößte Tageszeitung, der Staatsoperette einen ganzseitigen Artikel und prognostizierte einen Skandal ${ }^{(49)}$. So auch die Arbeiter Zeitung, die einen Monat später über die Entstehung einer „Operettentragikomödie“ über die politischen Wirren der Ersten Republik berichtete, „die sicher viel Staub aufwirbeln [würde]“(50). Die eigentliche Empörungswelle setzte an, als erste Bilder der

\footnotetext{
45 Zit. nach: Typoskript Novotnys, LhW/Dst/N1.29-3, Karton „Staatsoperette: Rezension, Prozess“, Mappe „Staatsbegräbnis - Staatsoperette“.

46 Zit. nach $e b d$.

47 Interview Novotnys, Wien, 15.12.2011.

48 F. Novotny, „Aus dem Staatsoperetten Nachrichtenstudio“, LhW/Dst/N1.29-3, Karton „Staatsoperette: Rezension, Prozess".

49 G. Pistor, „Mühsame Geburt der TV-Operette“ (Anm. 20).

50 „Die Erste Republik als Operette“, Arbeiter Zeitung, 4.3.1977, S. 14.
} 
Produktion veröffentlicht wurden, und zwar nicht von ungefähr ausgerechnet die pikante Darstellung des Prälaten "Schwarz" umgeben von barbusigen Damen anlässlich seines Besuches bei „Muffolini“ oder die karikaturhafte Inszenierung Dollfuß” als Zirkusfigur. Exemplarisch dafür sind die drei großformatigen Bilder, die im Wiener Wochenblatt vom 6. März 1977 unter dem reißerischen Titel „Dollfuß, Duce, Popscherl, Busen“ veröffentlicht wurden. Indem sie die Aufmerksamkeit des Publikums auf einzelne, gewagte Szenen lenkte und den Film auf ein paar nicht-repräsentative Bilder reduzierte, trug die visuelle Berichterstattung wesentlich zur öffentlichen Schmähung des Films bei.

Ursprünglich hätte die Staatsoperette am 26. Oktober, dem österreichischen Nationalfeiertag, ausgestrahlt werden sollen. Unter dem Druck der Medien, der politischen Opposition und insbesondere des ORF-Kurators Heribert Steinbauer (ÖVP) wurde die Ausstrahlung jedoch um einen Monat verschoben, und dafür eine ORF-interne Vorführung organisiert. Unmittelbar nach dieser Vorführung brachten die ÖVP-Abgeordneten Josef Gruber, Heribert Steinbauer, Eduard Moser und Alois Leitner eine parlamentarische Anfrage ein, in der sie nach Möglichkeiten fragten, wie „die Förderung derartiger Filme“ - Leitner sprach später von „Anti-Kultur“(51) - künftig vermieden werden könne ${ }^{(52)}$. Die über die interne Premiere gewonnenen Einblicke trugen zudem wesentlich zur Verschärfung des medialen Sturms gegen den Film bei. In der Kleinen Zeitung vom 22. Oktober 1977 bezeichnete etwa der für seine Kontroversen und Provokationen bekannte katholische Autor und Kronen-Zeitung-Kolumnist Kurt Dieman die Staatsoperette - obwohl er den Film zu diesem Zeitpunkt nur bestenfalls aus zweiter Hand kennen konnte - als einen „Anschlag auf das Staats- und Geschichtsbewußtsein jedes anständigen Österreichers“(53). Solche Vorwürfe wurden dann rasch in etlichen Tagesmedien bis hin zu lokalen Kleinblättern übernommen und somit buchstäblich flächendeckend verbreitet. Die Hetzkampagne ging so weit, dass die LeserInnen unmittelbar vor der Ausstrahlung am 30. November 1977 ausdrücklich zum aktiven Widerstand gegen den Film aufgefordert wurden. „Was halten Sie davon?“, fragte die Kleine Zeitung ihre Leser am Tag der Ausstrahlung, und forderte sie auf, ihre Meinung telephonisch an den ORF zu übermitteln ${ }^{(54)}$. „Umstrittene ,Staatsoperette heute in FS2: Schreiben Sie uns Ihre Meinung!“, bot ebenfalls die Kronen Zeitung an ${ }^{(55)}$.

Allein während der Ausstrahlung des Films registrierte die Kundendienststelle des ORF 647 Anrufe - davon 43 positive und 598 negative Stellungnahmen ${ }^{(56)}$. Die vom

51 Stenographisches Protokoll der 75. Sitzung des Nationalrates der Republik Österreich, XIV. Gesetzgebungsperiode, 6.12.1977, S. 7220 .

52 Stenographisches Protokoll der 70. Sitzung des Nationalrates der Republik Österreich, XIV. Gesetzgebungsperiode, 17.11.1977, S. 6660.

53 Kurt Dieman, „,Staatsoperette': Staatsskandal“, Kleine Zeitung, 22.10.1977, LhW/Dst/N1.29-3, Karton „Staatsoperette: Rezension, Prozess“, Mappe „Staatsbegräbnis - Staatsoperette“.

54 „Was halten Sie davon?“, Kleine Zeitung Graz, 30.11.1977, LhW/Dst/N1.29-3, Karton „Staatsoperette: Rezension, Prozess“, Mappe „Rezensionen“.

55 Kronen-Zeitung, 30.11.1977, LhW/Dst/N1.29-3, Karton „Staatsoperette: Rezension, Prozess“, Mappe „Staatsoperette ORF-Dokumentation“.

56 Laut dem Kurier meldete die Kundendienststelle auch am nächsten Tag mehr als 1200 Anrufe. Vgl.: „Staatsoperette: Negativ-Rekord“, Kurier, 7.12.1977, LhW/Dst/N1.29-3, Karton „Staatsoperette: Rezension, Prozess“, Mappe „Staatsoperette ORF-Dokumentation“. 
Kundendienst protokollierten, kritischen Urteile reichten von: „Den Verantwortlichen dafür sollte man aufhängen“, über: „So geschmacklos war der ORF noch nie“; „so etwas wäre nicht einmal im kommunistischen Osten möglich“; „den dafür Verantwortlichen hat man offensichtlich ins Hirn geschissen“ bis hin zu: „Mit so einer Hetzsendung züchtet man nur einen zweiten Hitler“ oder eben: „Unter Hitler hätte es so etwas nicht gegeben “(57).

Parallel dazu denunzierten die konservativen Kritiker den Umstand, dass es sich bei diesem provokativen Film um eine Fernsehproduktion des öffentlichen Rundfunks handelte, und interpretierten die scharfe Parodierung des katholisch-konservativen Lagers als regierungstreue Propaganda. So bemerkte Dieman: „Es ist sehr fraglich, ob man auch bereit gewesen wäre, eine derart respektlose und geschmacklose Parodie über führende sozialistische Persönlichkeiten durchgehen zu lassen “(58). Wie an diesem Zitat bereits ersichtlich wird, wurde der Vorwurf der Parteilichkeit, und darüber hinaus die Kritik an dem Film zum willkommenen Anlass für die Delegitimierung der Regierung Kreisky und insbesondere deren jüngster Rundfunkreform, die aus dem ORF eine öffentlich-rechtliche Institution machte ${ }^{(59)}$. In diesem Sinne kolportierte die ÖVPnahe Salzburger Volkszeitung am Tag der Ausstrahlung: „Die Sendung ,Staatsoperette spiegelt nach Ansicht [des konservativen Abgeordneten, Anm. LD] Steinbauers in eindrucksvoller Weise den Zustand des gegenwärtigen Regierungsrundfunks wider und macht den gebührenzahlenden Fernsehteilnehmern klar, wie ihr Geld verschwendet wird“(60). Der Kontext der 1970er Jahre veranlasste darüber hinaus viele KritikerInnen, den Film als neue Form des Terrorismus und die fördernde Regierung als dessen Unterstützerin zu brandmarken. So wurde etwa der Film am 30. November und 1. Dezember Teil einer Parlamentsdebatte rund um die „dringliche Anfrage“ der ÖVP betreffend eine mangelnde Vorsorge für die Terrorbekämpfung in Österreich ${ }^{(61)}$. In den nächsten Tagen fanden sich diese Argumente auch in den Medien wieder. So schrieb die Kleine Zeitung vom 2. Dezember, dass das Land durch die Subventionierung der Staatsoperette der „Terrorszene, die sich auch in Österreich schon aufbaut, ein beträchtliches Stück nähergerückt“ sei, und warf den Förderstellen vor, „sich mitschuldig an der Rechtfertigung des Terrors von gestern und der Vorbereitung des Terrors von morgen“ zu machen ${ }^{(62)}$.

Bei der Verurteilung des Films als Propagandainstrument der sozialistischen Regierung ließen die konservativen Kritiker außer Acht, dass die Staatsoperette, so scharf sie in der Tat gegen die Geschichtsverklärung des katholisch-konservativen Lagers

57 Zit. nach: LhW/Dst/N1.29-3, Karton „Staatsoperette: Rezension, Prozess“, Mappe „GÖA-Kundendienst - Genaue telefonische Reaktionen: Donnerstag, 30. November 1977“.

58 Interview Diemans in: „Staatsoperette - ein geschmackloses Pamphlet“, Süd-Ost Tagespost, 30.11.1977, LhW/Dst/N1.29-3, Karton „Staatsoperette: Rezension, Prozess“.

59 Dementsprechend bezeichnete Dieman den Film als „die erschreckendste Folge der Rundfunkreform Kreiskys \& Benyas, Brodas \&Blechas“. Vgl. K. Dieman, „Staatsoperette“ (Anm. 53).

60 Zeitungsnotiz, in: Salzburger Volkszeitung, 30.11.1977, LhW/Dst/N1.29-3, Karton „Staatsoperette: Rezension, Prozess“, Mappe „Rezensionen“.

61 Vgl. Stenographisches Protokoll der 72. Sitzung des Nationalrates der Republik Österreich, XIV. Gesetzgebungsperiode, 1.12.1977, S. 6831ff.

62 Fritz Csoklich, „Die Lust, alte Gräber neu auszuheben“, Kleine Zeitung, 2.12.1977, LhW/Dst/N1.29-3, Karton „Staatsoperette: Rezension, Prozess“, Mappe „Rezensionen“. 
vorging, alles andere als zimperlich mit der Sozialdemokratie umging: sei es über die gnadenlose Karikatur ihres „Säulenheiligen“ Otto Bauer und die Parodierung der folgenschweren Diskrepanz zwischen Verbalradikalismus und Attentismus in der Sozialdemokratie der 1920er und 1930er Jahre, oder über die regelrechte Denunzierung der rückständigen Kulturpolitik der roten Regierung der 1970er Jahre in den Rahmensequenzen. Die geringe Reaktion der sozialdemokratischen Partei auf diese Komponente der Satire mag darauf zurückzuführen sein, dass die im Film vorkommende Kritik an den geschichtspolitischen Tabus der Sozialdemokratie und den Unzulänglichkeiten ihrer Kulturpolitik in der Rezeption sofort von den konservativen Vorwürfen gegen die Parodierung Seipels und Dollfuß’ überdeckt wurde und daher ohnehin nicht mehr „skandalisiert“ werden musste.

Neben der Terrorismus-Keule gehörten auch Geschmacklosigkeit, Pietätlosigkeit und Faktenwidrigkeit zu den häufigsten konservativen Vorwürfen gegen den Film. So sprach der damalige Pressereferent der Erzdiözese Wien, Erich Leitenberger, in Anlehnung an den konservativen ORF-Kuratoren Eduard Ploier von einer „äußerst geschmacklosen, nicht verantwortbaren Verfälschung der geschichtlichen Situation“ und kritisierte weiter: „Offensichtlich ist man in manchen Kreisen der, linken Reichshälfte auch nach vier Jahrzehnten noch nicht bereit, einem Seipel oder einem Dollfuß ein Mindestmaß an Gerechtigkeit widerfahren zu lassen “(63). Ebenfalls mit Hinweis auf dieses Mindestmaß an Gerechtigkeit rang sich die damals noch fundamentalkatholische Zeitung Die Furche sogar dazu durch, besondere Qualitäten des faschistischen und kirchenfeindlichen Führers Mussolini zu unterstreichen: „Nur war Mussolini zweifellos ein Gangster, hatte aber doch wohl etwas mehr Format als seine roten diktatorischen Nachahmer und Epigonen. Die elegantesten und interessantesten Frauen Italiens liefen ihm nach und eine von ihnen war bereit, mit dem Duce in den Tod zu gehen“ “(64).

Einer der Kritikpunkte, die Novotny und Zykan besonders getroffen zu haben scheinen, ist die Gleichsetzung dieser Satire und insbesondere der Parodierung Dollfuß' mit propagandistischen Maßnahmen der Nationalsozialisten, wie etwa in ihren derben Dollfußkarikaturen. So klagte eine empörte Zuschauerin in einem Brief an Zykan an:

„Und Sie [...] finden es für richtig, Dr. Dollfuß wegen seines kleinen Körperwuchses als bösartigen Zwerg vor Millionen Zuschauern in diesem Land zur Verhöhnung und Verspottung herumradeln zu lassen. Diese Bösartigkeit, Perfidie und sadistische Dummheit ist nicht mehr zu überbieten. Sind wir schon wieder soweit, daß Menschen wegen ihrer körperlichen Besonderheiten oder Behinderungen öffentlich verspottet werden können, so wie zur Zeit des ,Stürmers' oder des ,Unwerten Lebens “ “(65).

Entgegen solcher Vorwürfe hielt Novotny fest, dass der Witz immer schon eine legitime Waffe gewesen sei, und dass Satire daher nicht an sich pauschal verurteilbar sei,

63 Erich Leitenberger, „Geschmacklose ,Staatsoperette“, Präsent, Österreichische Wochenzeitung, 3.11.1977, LhW/Dst/N1.29-3, Karton „Staatsoperette: Rezension, Prozess“, Mappe „Staatsbegräbnis - Staatsoperette“.

64 Erich Thanner, „Österreich als Staatsoperette“, Die Furche, 16.09.1977, LhW/Dst/N1.29-3, Karton „Staatsoperette: Rezension, Prozess“, Mappe „Staatsoperette ORF-Dokumentation“.

65 Berta Pirker, Brief an Otto M. Zykan, 1.12.1977, LhW/Dst/N1.29-3, Karton „Staatsoperette: Rezension, Prozess“, Mappe „Staatsbegräbnis - Staatsoperette“. 
sondern an ihrer politischen Zielsetzung bemessen werden sollte - und vor diesem Hintergrund sei eine Gleichsetzung der Staatsoperette mit NS-Propaganda unzulässig ${ }^{(66)}$.

Die Hetzkampagne rief aber auch FürsprecherInnen auf den Plan. In diesem Sinne sprach etwa die kommunistische Zeitung Wahrheit am Tag der Ausstrahlung von einem „programmierten ,Skandal ““(67). In derselben Zeitung rief der kommunistische Lyriker und Journalist Arthur West ein paar Tage später dazu auf, die „Kläger [...] anzuklagen“, und kritisierte dabei in erster Linie die konservative Vorherrschaft im Kulturbereich:

„Nach dem kläglichen Scheitern der reaktionären Kampagne gegen die ,Alpensaga brauchte ,man' ein neues Opfer. Und fand es prompt. Und organisierte eine Aktion bösester, gefährlichster Diffamierung - nein, nicht nur, ja nicht eigentlich gegen die Autoren der ,Staatsoperette und gegen den angeblich so ,roten' ORF, sondern schlechtweg gegen alles, was da gegen die herrschenden Machtstrukturen und ihre Verdummungsindustrie aufzumucken wagt“" (68).

Auch Elfriede Jelinek beklagte nach der Ausstrahlung die rückschrittliche Kulturpolitik Österreichs, betonte aber vor allem die Schuld der Sozialdemokratie an dieser Situation, indem sie festhielt: „Die ,Staatsoperette hat mir sehr gefallen, nicht zuletzt wegen der bösartigen Kritik an der Sozialdemokratie, die ja zum Beispiel kulturell mehr vernichtet hat als selbst konservative Richtungen. Das ist für mich ganz übel“ (69).

Die kulturpolitischen Hauptfolgen des Filmes bekam allerdings Novotny zu spüren, dessen Karriere als Regisseur des ORF nach dem Skandal um den Film de facto beendet war. Nichtsdestotrotz hatte der Film insofern unmittelbare gesellschafts- und geschichtspolitische Konsequenzen, als er eine öffentliche Auseinandersetzung mit dem Stand des Geschichtsunterrichts und darüber hinaus des Geschichtsbewusstseins in Österreich in die Wege leitete. So rechtfertigte etwa der sozialistische Unterrichtsminister Fred Sinowatz die öffentliche Subventionierung des Films gegen die Kritiker der ÖVP mit dem Argument: „Von den Herstellern des Filmes wollte man offensichtlich einen Denkund Diskussionsanstoß im Hinblick auf die neuere Geschichte Österreichs geben und es ermöglichen, sich kritisch mit geschichtlichen Vorgängen auseinanderzusetzen “ (70). Vor diesem Hintergrund mag die durch die Staatsoperette ermöglichte Debatte über die Lücken des Geschichts- und Demokratiebewusstseins in Österreich auch zur endgültigen Implementierung von Sinowatz' sogenanntem „Grundsatzerlass“ zur Politischen Bildung im April 1978 beigetragen haben, wodurch die Politische Bildung, verstanden als „Erziehung zu einem demokratischen Österreichbewusstsein“, als Unterrichtsprinzip in den österreichischen Schulen etabliert wurde ${ }^{(71)}$.

66 Vgl. Typoskript Novotnys, LhW/Dst/N1.29-3, Karton „Staatsoperette: Rezension, Prozess“, Mappe „Staatsbegräbnis - Staatsoperette“.

67 „Programmierter,Skandal““, Wahrheit, 30.11.1977, LhW/Dst/N1.29-3, Karton „Staatsoperette: Rezension, Prozess“, Mappe „Rezensionen“.

68 Arthur West, „Die Kläger sind anzuklagen“, Wahrheit, 2.12.1977, LhW/Dst/N1.29-3, Karton „Staatsoperette: Rezension, Prozess“, Mappe „Rezensionen“.

69 Elfriede JelineK, in: Volksstimme, 15.10.1982, zit. nach I. Suchy, „Das Werden der Staatsoperette in Originaldokumenten“ (Anm. 17), S. 90.

70 Zit. nach ebd., S. 99.

71 Grundsatzerlass „Politische Bildung“, zit. nach Rupert Rappersberger, Alexander Nirgowics, Politische Bildung in Österreich. Historische Betrachtung und empirische Studie, Dipl.-Arb., Wien, 2010, S. 183. 


\section{Schluss}

Abschließend scheint es angebracht zu betonen, dass die Staatsoperette, abgesehen von dem Skandal, den sie auslöste und der seitdem mit ihrem Namen assoziiert ist, in erster Linie ein avantgardistisches, vielschichtiges und aufwendiges Kunstwerk darstellte. Dieser Experimentcharakter zeigt sich bereits in den von der bisherigen Forschung unterbelichteten Rahmensequenzen, über welche die Künstler die geschichtspolitische Satire um eine kulturpolitische Dimension ergänzten. Noch innovativer als diese unübliche zeitliche Auffächerung des Erzählformats war wohl die Gestaltung des Hauptteils des Films, in der Novotny und Zykan die Tradition der rührseligen Wiener Operette absichtlich verfremdeten, um sie zu den ursprünglichen gesellschaftskritischen Ansprüchen dieses musiktheatralischen Genres zurückzuführen. Die über diese experimentelle Operette dargebotene Satire bestand nicht nur in einer Parodierung der historischen Hauptprotagonisten der Zwischenkriegszeit, sondern bediente sich auch eines komplexen Abstrahierungs- und Verdichtungsverfahrens, das dazu diente, historische Begebenheiten und Figuren in parabelhafte Anspielungen zu verwandeln, um damit das vorherrschende Geschichtsbild zur Debatte zu stellen. An dieser Stelle stolperten die Künstler jedoch über ihre eigene Kreativität: Die hohen künstlerischen Ansprüche überstiegen das Rezeptionspotential des breiten Publikums und erwirkten sich damit negativ auf das aufklärerische Potential des Filmes aus. So scheiterte der angestrebte subversive Beitrag zur gesellschaftlichen Aufarbeitung der tabuisierten Zeitgeschichte bis zum einem gewissen Grad daran, dass es nur von einem eingeweihten Elitenpublikum verstanden werden konnte. In dieser Diskrepanz zwischen künstlerischem und gesellschaftspolitischem Anspruch liegt, aus geschichtspolitischer Perspektive, der heikelste Punkt des gesamten Werks.

\section{Quellen- und Literaturverzeichnis}

Bотz Gerhard, „Die Ausschaltung des Nationalrates und die Anfänge der Diktatur Dollfuß’ im Urteil der Geschichtsschreibung von 1933 bis 1973“, in: Anton BENYA (Hg.), Vierzig Jahre danach. Der 4. März 1933 im Urteil von Zeitgenossen und Historikern, Wien, Doktor-KarlRenner-Institut, 1973, S. 31-59.

Вотz Gerhard, Gewalt in der Politik. Attentate, Zusammenstöße, Putschversuche, Unruhen in Österreich 1918 bis 1938, München, Wilhelm Fink Verlag, 1983.

Chaplin Charles, My autobiography, New York, Simon and Schuster, 1964.

Gratzer Wolfgang, „Die Staatsoperette, ein signifikanter Skandal“, in: Peter Csobádi, Gernot Gruber (u. a.) (Hg.), Politische Mythen und nationale Identitäten im (Musik-)Theater, Anif/ Salzburg, Mueller-Speiser Verlag, 2003, Bd. II, S. 890-903.

Hanisch Ernst, „Die Dominanz des Staates. Österreichische Zeitgeschichte im Drehkreuz von Politik und Wissenschaft“, in: Alexander Nützenadel, Wolfgang Schieder (Hg.), Zeitgeschichte als Problem. Nationale Traditionen und Perspektiven der Forschung in Europa, Göttingen, Vandenhoeck \& Ruprecht, 2004, S. 54-77.

Hantsch Hugo, „Engelbert Dollfuß (1892-1934)“, in: Ders. (Hg.), Gestalter der Geschicke Österreichs, Innsbruck/Wien/München, Tyrolia-Verlag, 1962, S. 611-623.

Interview mit Franz Novotny, Wien, 15.12.2011 (Aufzeichnungen im Besitz der Autorin).

Jedlicka Ludwig, Rudolf Neck (Hg.), Vom Justizpalast zum Heldenplatz, Studien und Dokumentationen 1927 bis 1938, Wien, Verlag der Österreichischen Staatsdruckerei, 1975.

Literaturhaus Wien, Dokumentationsstelle für neuere österreichische Literatur, Dst-Handschriftensammlung, Vorlass Franz Novotny, N1.29-3. 
Polt-Heinzl Evelyne, „Kulturskandale der 1970er Jahre. Lauter kleine Staatsoperetten“, in: Ders. (Hg.), Staatsoperetten. Kunstverstörungen. Das kulturelle Klima der 1970er Jahre, Wien, Dokumentationsstelle für Neuere Österreichische Literatur, 2009, S. 9-42.

Rappersberger Rupert, Alexander Nirgowics, Politische Bildung in Österreich. Historische Betrachtung und empirische Studie, Dipl.-Arb., Wien, 2010.

Stenographische Protokolle des Nationalrates der Republik Österreich, XIII. und XIV. Gesetzgebungsperiode.

Suchy Irene, „Das Werden der Staatsoperette in Originaldokumenten. Rekonstruktion eines Prozesses“, in: Evelyne Polt-Heinzl (Hg.), Staatsoperetten. Kunstverstörungen. Das kulturelle Klima der 1970er Jahre, Wien, Dokumentationsstelle für Neuere Österreichische Literatur, 2009, S. 86-122.

ZELGER Sabine, „Die Störung des nationalen Blicks oder Warum der österreichische Bildungskanon nicht auf die Staatsoperette verzichten darf“, in: Evelyne Polt-Heinzl (Hg.), Staatsoperetten. Kunstverstörungen. Das kulturelle Klima der 1970er Jahre, Wien, Dokumentationsstelle für Neuere Österreichische Literatur, 2009, S. 123-134.

\section{Zusammenfassung}

„Gottlose Propaganda“, „unverschämte Verfälschung der Geschichte“, „Pornographie“, „intellektualistischer Terrorismus“ - von solchen Anklagen und vielen mehr wurde die Ausstrahlung des Films Staatsoperette auf Österreichs öffentlichem Fernsehsender FS 2 am 30. November 1977 begleitet. Die Rahmengeschichte des Films handelt von den Schwierigkeiten eines jungen Regisseurs bei der Gestaltung eines Films im verkrusteten kulturpolitischen Kontext der 1970er Jahre in Österreich. Auf einer zweiten Ebene spielt die Kernhandlung des Films, die den eigentlichen Skandal auslöste: eine musikalische Tragikomödie, die mittels Satire und schwarzen Humors die großkoalitionär geprägten Mythen und Tabus rund um die diktatorische Wende der 1930er Jahre in einer präzedenzlosen Schärfe angreift. Nach einer Einbettung des avantgardistischen Filmprojekts in den politischen Kontext seiner Entstehung analysiert der Beitrag die politischen Motivationen der Künstler sowie ihre Umsetzungstechniken, liefert einen Einblick in den Skandal und seine Hintergründe, und geht abschließend der Frage der längerfristigen Wirkung und Bedeutung des Films nach.

\section{Résumé}

"Propagande impie ", "honteuse falsification de l'histoire ", "pornographie ", "terrorisme intellectualiste!»-autant de qualificatifs critiques que le film scandale Staatsoperette recueillit dans l'opinion publique autrichienne à sa diffusion le 30 novembre 1977 sur la chaîne nationale. Le cadre de l'histoire se situe dans l'Autriche des années 1970 et met en scène un dramaturge confronté à de nombreux obstacles lors de la création d'un film jugé trop politisé par le monde de la culture sclérosé de son époque. Au second plan, qui devient le centre du film, on découvre l'objet de la critique : une tragicomédie musicale dénonçant par la satire et l'humour noir l'écriture de l'histoire "coalitionnaire " autrichienne et notamment les mythes et tabous entourant le tournant dictatorial des années 1930 en Autriche. Après avoir resitué l'ouvre dans le contexte politique des années 1970, cette contribution analyse les techniques filmiques et les motivations politiques des artistes, avant de revenir sur le scandale provoqué par le film et sur sa portée artistique et politique à plus long terme. 